Technological University Dublin

DÜBLIN

ARROW@TU Dublin

2008-09-01

\title{
A Technique for Characterization of Dimensional Changes in Slanted Holographic Gratings by Monitoring the Angular Selectivity Profile
}

\author{
Nitesh Pandey \\ Technological University Dublin \\ Izabela Naydenova \\ Technological University of Dublin, izabela.naydenova@tudublin.ie \\ Suzanne Martin \\ Technological University of Dublin, suzanne.martin@tudublin.ie
}

See next page for additional authors

Follow this and additional works at: https://arrow.tudublin.ie/cieoart

\section{Recommended Citation}

Pandey, N. et al. (2008) Technique for characterization of dimensional changes in slanted holographic gratings by monitoring the angular selectivity profile. Optics Letters, Vol. 33, No. 17, September. doi:10.1364/OL.33.001981

This Article is brought to you for free and open access by the Centre for Industrial and Engineering Optics at ARROW@TU Dublin. It has been accepted for inclusion in Articles by an authorized administrator of ARROW@TU Dublin. For more information, please contact arrow.admin@tudublin.ie, aisling.coyne@tudublin.ie, gerard.connolly@tudublin.ie.

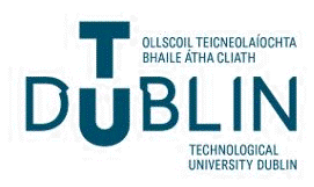




\section{Authors}

Nitesh Pandey, Izabela Naydenova, Suzanne Martin, and Vincent Toal 


\title{
A technique for characterization of dimensional changes in slanted holographic gratings by monitoring the angular selectivity profile
}

\author{
Nitesh Pandey, Izabela Naydenova, Suzanne Martin, Vincent Toal \\ Centre for Industrial and Engineering Optics, Dublin Institute of Technology, Kevin Street, \\ Dublin 8, Dublin, Ireland \\ *Corresponding author: nitesh.pandey@student.dit.ie
}

A method has been developed for retrieving the complete angular selectivity profile of transmission holographic gratings in one step. The method is applied to study in real time the shrinkage and changes in the effective optical thickness of a slanted holographic grating recorded in an acrylamide based photopolymer. It can form the basis of a holographic sensor of analytes which cause a thickness change in the holographic medium. It can also be useful for the study and optimization of holographic recording materials and for quality control during production of holographic optical elements

OCIS codes: 090.0090, 090.7330, 090.2890, 130.6010, 160.2900, 160.5335

Volume holographic gratings recorded in photopolymers find use in applications such as grating filters, holographic interconnects, optical elements[1] and holographic data storage[2], to name a few. One of the key factors affecting the performance of such gratings is the sensitivity of the dimensions of the medium to temperature, humidity, and other environmental changes. Shrinkage of photopolymers [3] has always been an important consideration in holographic data storage and temperature induced changes in the holographic medium can significantly affect the fidelity of the reconstruction in holographic data storage [4]. However, this dimensional dependence can also be useful in making spectral filters [5] and humidity sensors[6]. Real time monitoring of the photopolymer shrinkage is very useful as it can give us information on the polymerization rate, extent of detuning and change in optical thickness. In the present paper we demonstrate a method that can be used to study dimensional changes in slanted phase holographic grating in real time. Consider a slanted transmission holographic grating (Fig 1) of thickness $\mathrm{L}$ with a slant angle $\varphi, \varepsilon_{l}$ is the modulation of the permittivity $\varepsilon_{0}$ of the material. The diffraction efficiency [7] of this transmission hologram for a plane wave incident at an angle $\Delta \theta$ away from $\theta$ is given by

$\eta(\Delta \theta)=\left(\frac{\varepsilon_{1} k L}{2 \varepsilon_{0} \cos \theta}\right)^{2} \sin c^{2}\left(\frac{2 L(\Delta \theta) \sin \theta}{\lambda}\right),(1)$

where $k$ is $2 \pi / \lambda$ and $\lambda$ is the wavelength. Equation (1) is the angular selectivity profile of the grating and is usually obtained by rotating the plane of the grating and measuring the diffracted intensity for different incident angles. Consider the situation in which a thick transmission holographic grating is illuminated with a probe beam having a spherical wavefront with an angular spread of $\Delta \theta<<\theta$ around the the Bragg angle $\theta$. If the grating is placed a distance $d$ from the focus of a lens (Fig. 2(a)) such that the Bragg matched component meets the grating at a distance $x$, then the angular detuning $\Delta \theta_{d x}$ corresponding to a distance $x+d x$, is $\Delta \theta_{d x}=d x \cdot \cos (\theta) / d$ 
For thick gratings $(\mathrm{L}>100 \mu \mathrm{m})$, the angular selectivity is high and we assume that the intensity of the probing beam is constant over the range $\Delta \theta$ The intensity of the diffracted beam in the $X Y$ plane is given by

$$
\eta(x)=\left(\frac{\varepsilon_{1} k L}{2 \varepsilon_{0} \cos \theta}\right)^{2} \sin c^{2}\left(\frac{2 L(x \cos \theta) \sin \theta}{\lambda d}\right),(2)
$$

The resulting diffraction pattern (Fig 2(b)) is modulated in the $x$ direction and can be imaged on a CMOS sensor and the angular selectivity profile can be retrieved. If the shrinkage (or swelling) factor is $\alpha$, the recorded fringes rotate by an angle $\Delta \varphi$ which can be shown [8] to be $\Delta \varphi=\tan -1[\tan \varphi /(1+\alpha)]$ where $\phi$ is the initial slant angle. The Bragg angle will now be shifted by $\Delta \varphi$, which can be measured from the calibrated images. This gives us the capability of monitoring the angular selectivity of the growing gratings in real time. The FWHM of the curves can be retrieved and the changing optical thickness can also be monitored. We demonstrate the potential of our method in two ways. We monitor, in near real time, the Bragg detuning of a holographic grating while recording and evaluate the shrinkage of the material. Secondly we observe the effect of exposure to a high humidity environment on the dimensions of a prerecorded volume transmission grating. The photopolymer solution was prepared using acrylamide $(0.6 \mathrm{~g})$, bis-acrylamide $(0.2 \mathrm{~g})$, TEA $(2 \mathrm{ml})$ in a PVA stock solution $(10 \% \mathrm{wt})$. It was sensitized to recording at 532nm using the dye, Erythrosine B (4ml of $0.11 \%$ wt stock solution). Photopolymer layers were fabricated using gravity settling on $5 \times 5 \mathrm{~cm} 2$ glass plates and drying for 24 hours in a dark room. Light from a Nd-YVO 4 laser $(532 \mathrm{~nm})$ was spatially filtered, expanded and collimated and split into two s-polarized beams. These were made to interfere and a grating of spatial frequency 600 lines/mm was recorded having a slant angle of $15^{\circ}$ using intensity, 5 $\mathrm{mW} / \mathrm{cm}_{2}$ and exposure time $100 \mathrm{~s}$. The probe beam was a $633 \mathrm{~nm}$ He-Ne laser (Fig 2a). The grating was placed at a distance of $0.8 \mathrm{~cm}$ from the focus of an objective and the central lobe of the resulting diffraction pattern were captured using a 10 Megapixel CMOS sensor (Canon EOS 400D, pixel pitch $5.7 \mu \mathrm{m} \times 5.7 \mu \mathrm{m}$ ) with a resolution of 1960 pixels in the $x$ direction, placed at a distance of $32 \mathrm{~cm}$ from the sample. The profiles were retrieved at intervals of approximately 1 second. The retrieval time was 0.002 seconds. The images were smoothed using moving average to remove high frequency noise. To calibrate the experimental setup, a recorded grating was mounted on a high precision computer controlled rotational stage (Newport M-URM100ACC) and the Bragg profile was measured with an accuracy of $0.0001^{\circ}$ using a collimated probe beam. The FWHM of the profiles obtained by both methods were compared and it was seen that with the present geometry, a spread of 0.56 degrees corresponds to 700 pixels. This gives us a resolution of 0.0008 degrees per pixel. For thin gratings, the angular spread is larger and the profile of the probe beam must be taken into account. This can be done by capturing the intensity profile of the zero order beam and using the data to rescale the intensity profile of the diffracted light. In our case, rescaling does not significantly affect the original profile (Fig 2(d)) and was not required. Alternatively the Gaussian spread of the beam can be suitably reduced by beam expanding and filtering optics. In our case, the detuning of a single spatial frequency grating recorded using two plane wave beams covering a circular area of $1.5 \mathrm{~cm}$ diameter, is monitored. The spherical probe beam, generated using an objective ( $\mathrm{f}=15 \mathrm{~mm}, \mathrm{NA}=0.25)$ positioned with the grating at a distance of $0.8 \mathrm{~cm}$ from the focus, covers an area of diameter $0.2 \mathrm{~cm}$. Thus the total probing area is within this single grating. The grating is recorded in a vibration damped holographic setup. If the scheme is used to monitor gratings in shift multiplexing, the shift 
selectivity must be taken into account as the neighbouring gratings are a potential noise source. In this case, the shift selectivity is given by $\lambda z_{o} / L \sin \theta_{r}[9]$ where $\lambda$ is the recording wavelength, $z o$ is the distance from the focus, $L$ is the layer thickness and $\theta_{r}$ is the recording angle. In order to spherically probe such holograms, we must satisfy the selectivity constraint dictated by the recording geometry. So $\lambda_{p} . z p<\lambda_{r} . z r$ where $\lambda_{p}$ and $\lambda_{r}$ are the probing and recording wavelengths and $z p$ and $z r$ are distances from the probing and recording objectives. As shown in Fig. 3, after 100s the Bragg peak of the evolving grating is seen to detune by $0.11^{\circ}$, corresponding to $0.7 \%$ shrinkage in the photopolymer material. As the grating grew inside the medium, we observed that the FWHM of the profile decreased from $0.248^{\circ}$ at 11 s to $0.1928^{\circ}$ at 40s. The FWHM is dependent on the refractive index modulation, dye concentration and the variation of the refractive index modulation as a function of depth in the layer. The variation in these values causes the effective optical thickness to change. Using the relation[7] between the thickness and angular spread, $L=N 2 \Delta \theta \sin \left(\theta_{r}\right)$, an estimate can be made for the change: $d L / L=$ $d(\Delta \theta) / \Delta \theta=d(\mathrm{FWHM}) / \mathrm{FWHM}=0.0552 / 0.248=0.226(22.6 \%)$. This assumes that the refractive index modulation is uniform with depth, which is not the case. By modeling the photopolymerisation process[10] in combination with a rigorous coupled wave method $[11,12]$, the data obtained from these profiles can be used to compute the variation of the grating strength with time and depth in the photosensitive layer. On the other hand, shrinkage causes the fringes to reorient and causes a change in the position of the maximum in the diffracted beam. As the angular selectivity profile contains information about the position of the maximum and the FWHM , the method can detect and provide data for both. The experimental SNR is affected by the probe beam intensity, sensitivity of the camera, sensor noise and stray light into the sensor. Here however, for the single frequency grating, the detected profile is a well shaped sinc2(x) function, a fact which can be used for smoothing out noise. With proper processing (and even further curve fitting), the peak position can be detected with an accuracy corresponding to the pixel pitch of the sensor. In our case, for a slant angle of $10^{\circ}$, and pixel pitch of $5.7 \mu \mathrm{m}$, this corresponds to minimum detectable shrinkage of $0.02 \%$. Another application of the method is based on the fact that acrylamide/PVA based photopolymers are sensitive to humidity[6]. A 150 $\mu \mathrm{m}$ thick grating (slant angle $20^{\circ}$ ) and spacing of $1 \mu \mathrm{m}$ was recorded in the photopolymer, and the Bragg profile was obtained ('a' in Fig.4). The grating was then exposed to a high humidity environment (RH>90\%, 'b' in Fig 4) for less than 1 minute and the Bragg curve continuously monitored as the grating returned ('c' in Fig.4) to equilibrium with the $30 \%$ humidity environment. The graphs clearly demonstrate the shift in the Bragg peak as the grating shrinks due to the loss of absorbed moisture. The profile shifts by 194 pixels $\left(0.15^{\circ}\right.$ corresponding to $0.52 \%$ of the original thickness). In summary we have demonstrated a method for monitoring the shrinkage in photopolymers that can prove a useful tool for their optimization. We have further applied the method to sense dimensional changes in previously recorded slanted transmission gratings due to a change in the environment.

\section{Acknowledgments:}

This publication has emanated from research conducted with the financial support of Science Foundation Ireland.

\section{References}

1. Jacques Ludman, H John Caulfield, Juanita Riccobono,eds, Holography for the New Millennium, Springer, 2002 
2. Hans J.Coufal, Demetri Psaltis, Glenn T. Sincerbox, eds, Holographic data Storage,(Springer verlag, 2000)

3. W. S. Colburn and K. A. Haines, "Volume hologram formation in photopolymer materials," Appl. Opt. 10, 1636-1641 (1971)

4. Lisa Dhar, Melinda G. Schnoes, Theresa L. Wysocki, Harvey Bair, Marcia Schilling, and Carol Boyd , "Temperature-induced changes in photopolymer volume holograms", Appl. Phys. Lett. 73, 1337 (1998)

5. J. M. Russo and R. K. Kostuk, "Temperature dependence properties of holographic gratings in phenanthrenquinone doped poly(methyl methacrylate) photopolymers", Appl. Opt. 46, 74947499 (2007)

6. I.Naydenova, R. Jallapuram, V. Toal, and S. Martin, "A visual indication of environmental humidity using a color changing hologram recorded in a self-developing photopolymer," Appl. Phys. Lett. 92, 031109 ,(2008)

7. G.Barbastathis and D. Psaltis , "Volume Holographic Multiplexing Methods," in Holographic data Storage, Hans J.Coufal, Demetri Psaltis, Glenn T. Sincerbox, eds. (Springer verlag, 2000), pp. 21-62.

8. U.-S. Rhee, H. J. Caulfield, J. Shamir, C. S. Vikram, and M. M. Mirsalehi, "Characteristics of the DuPont photopolymer for angularly multiplexed page-oriented holographic memories," Opt. Eng. 32, 1839-1847 (1993).

9. G. Barbastathis, M. Levene, and D. Psaltis, "Shift multiplexing with spherical reference waves," Appl. Opt. 35, 2403-2417 (1996)

10. J. T. Sheridan and J. R. Lawrence, "Nonlocal-response diffusion model of holographic recording in photopolymer," J. Opt. Soc. Am. A 17, 1108-1114 (2000)

11. M. G. Moharam, E. B. Grann, D. A. Pommet, and T. K. Gaylord, "Formulation for stable and efficient implementation of the rigorous coupled-wave analysis of binary gratings," J. Opt. Soc. Am. A 12, 1068- (1995)

12. S. Gallego, M. Ortuño, C. Neipp, A. Márquez, A. Beléndez, I. Pascual, J. Kelly, and J. Sheridan, "Physical and effective optical thickness of holographic diffraction gratings recorded in photopolymers," Opt. Express 13, 1939-1947 (2005)

\section{Figure captions}

Fig.1 Probing a grating using a defocused beam. $\theta$ is the Bragg angle and $\Pi$ is the slant angle of the fringes. The intensity of the diffracted beam is modulated by the angular selectivity profile. Fig 2 a) Experimental setup b) Diffraction pattern on sensor. c) Raw profile retrieved by the CMOS array d) Smoothened profiles and zero order intensity (dotted curve) plotted before(dark curve) and after (light curve) rescaling.

Fig.3 The shift of the Bragg peak and corresponding shrinkage during exposure for 100s in a photopolymer grating. Insets show the changes in the profile width at $11 \mathrm{~s}, 23 \mathrm{~s}$ and $41 \mathrm{~s}$. Fig 4. Bragg detuning when exposed to high humidity. The thickness of the grating changes with the environmental humidity. 'a' is the original profile. 'b' is the high humidity profile, 'c' is the last measured profile. 


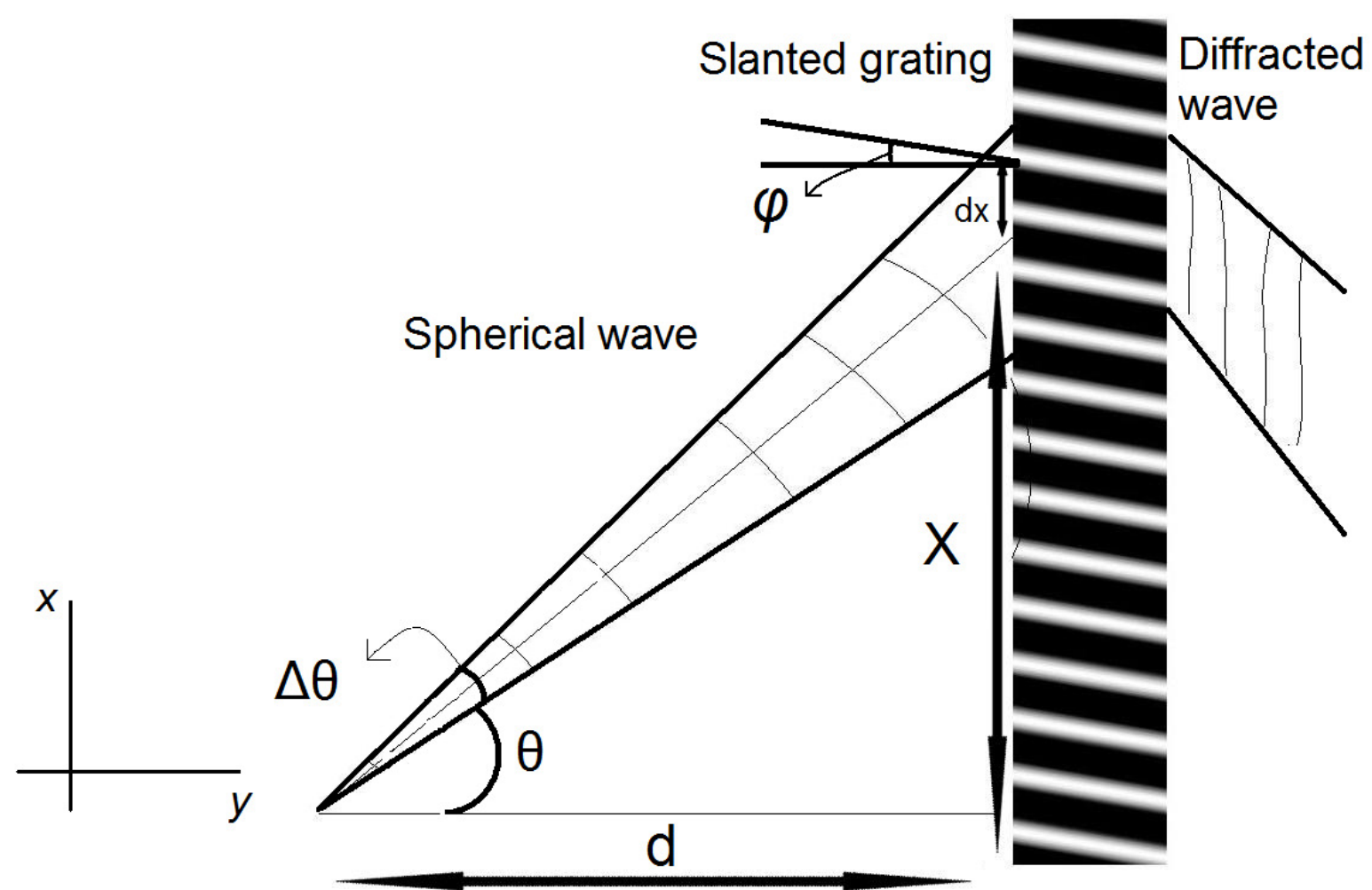

Fig.1 Probing a grating using a defocused beam. $\theta$ is the Bragg angle and $\Pi$ is the slant angle of the fringes. The intensity of the diffracted beam is modulated by the angular selectivity profile. 


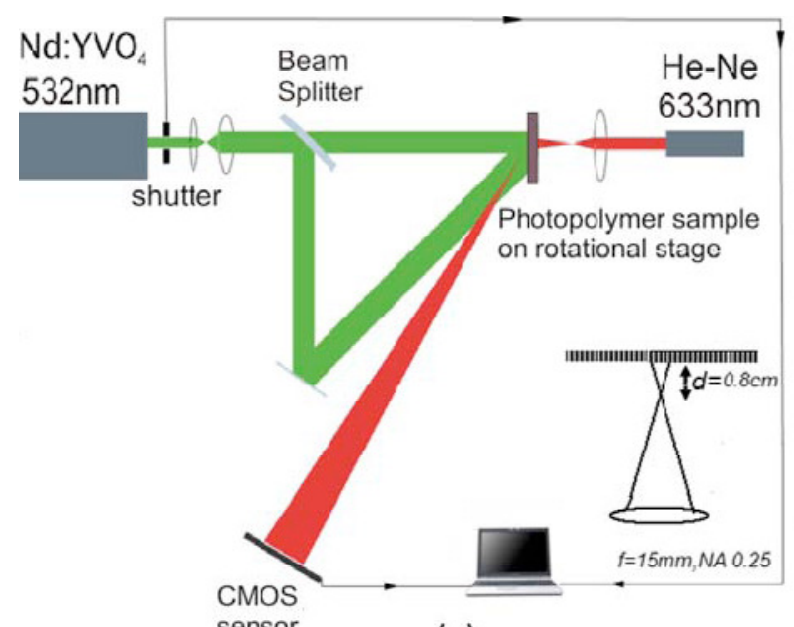

(a)

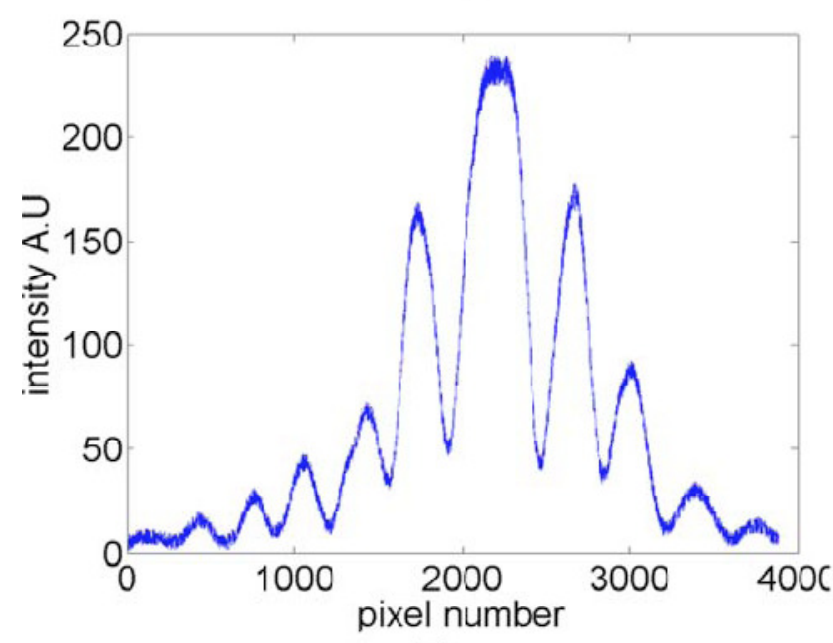

(c)

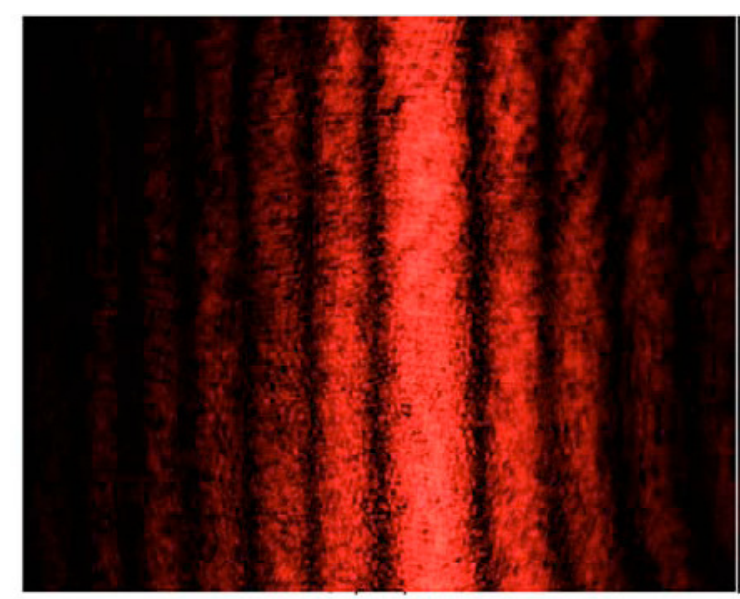

(b)

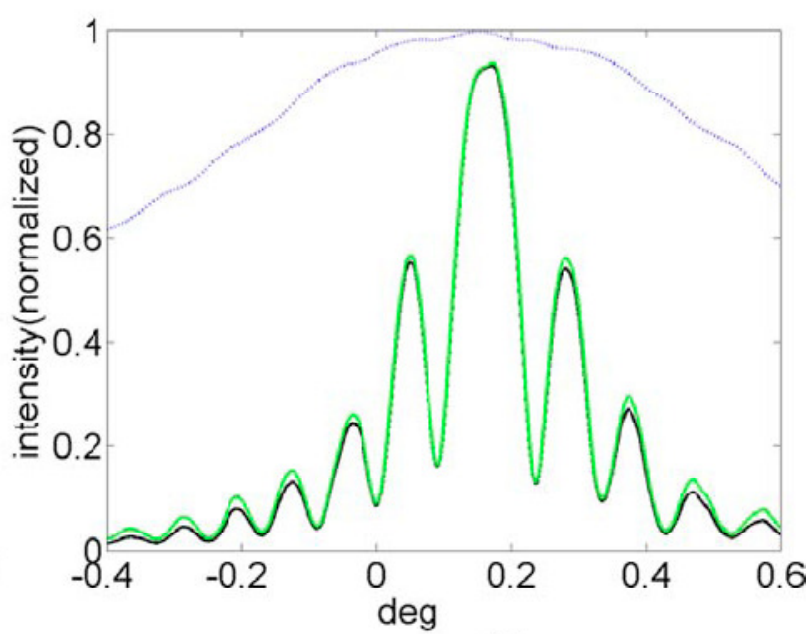

(d)

Fig 2 a) Experimental setup. b) Diffraction pattern on sensor. c) Raw profile retrieved by the CMOS array d) Smoothened profiles and zero order intensity (dotted curve) plotted before(dark curve) and after (light curve) rescaling. 


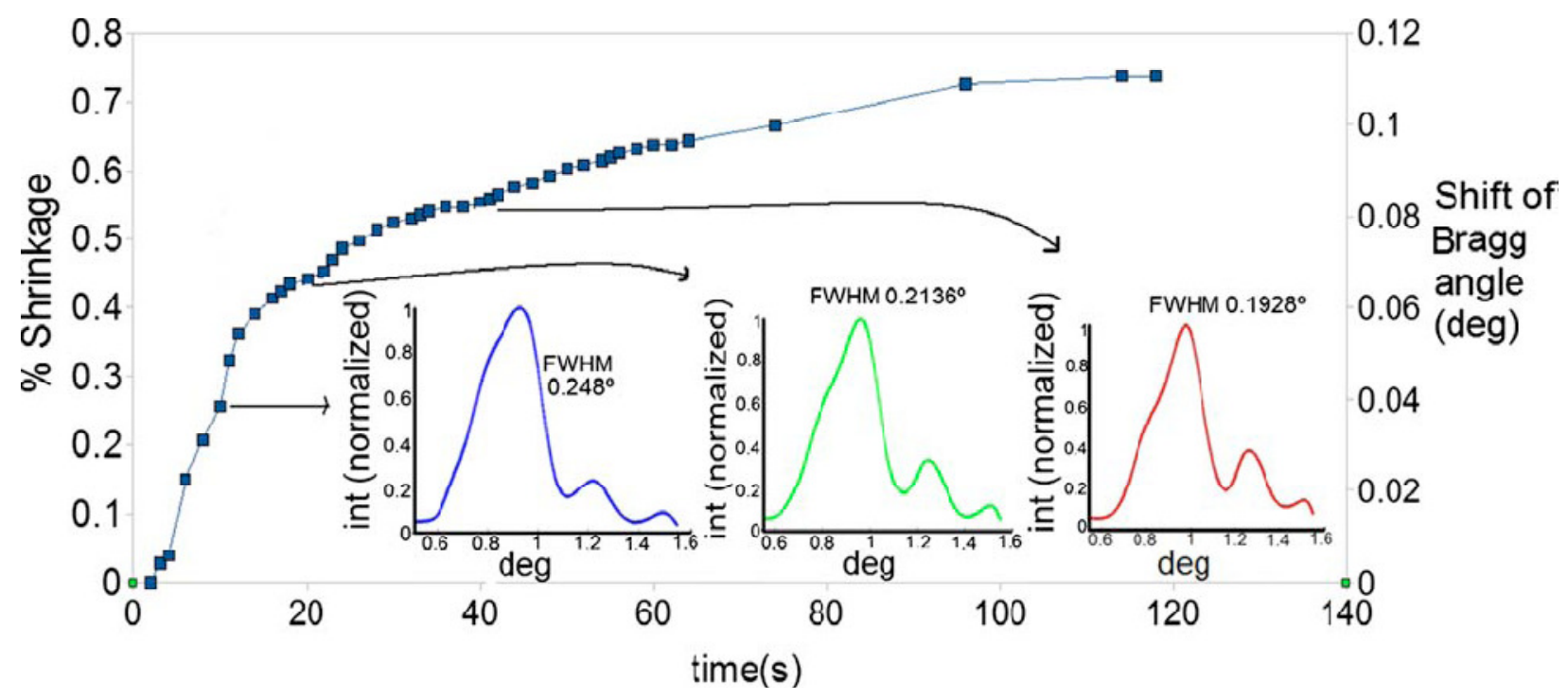

Fig.3 The shift of the Bragg peak and the corresponding shrinkage during exposure in a photopolymer grating. Insets show the changes in the profile width at $11 \mathrm{~s}, 23 \mathrm{~s}$ and $41 \mathrm{~s}$ during exposure. 


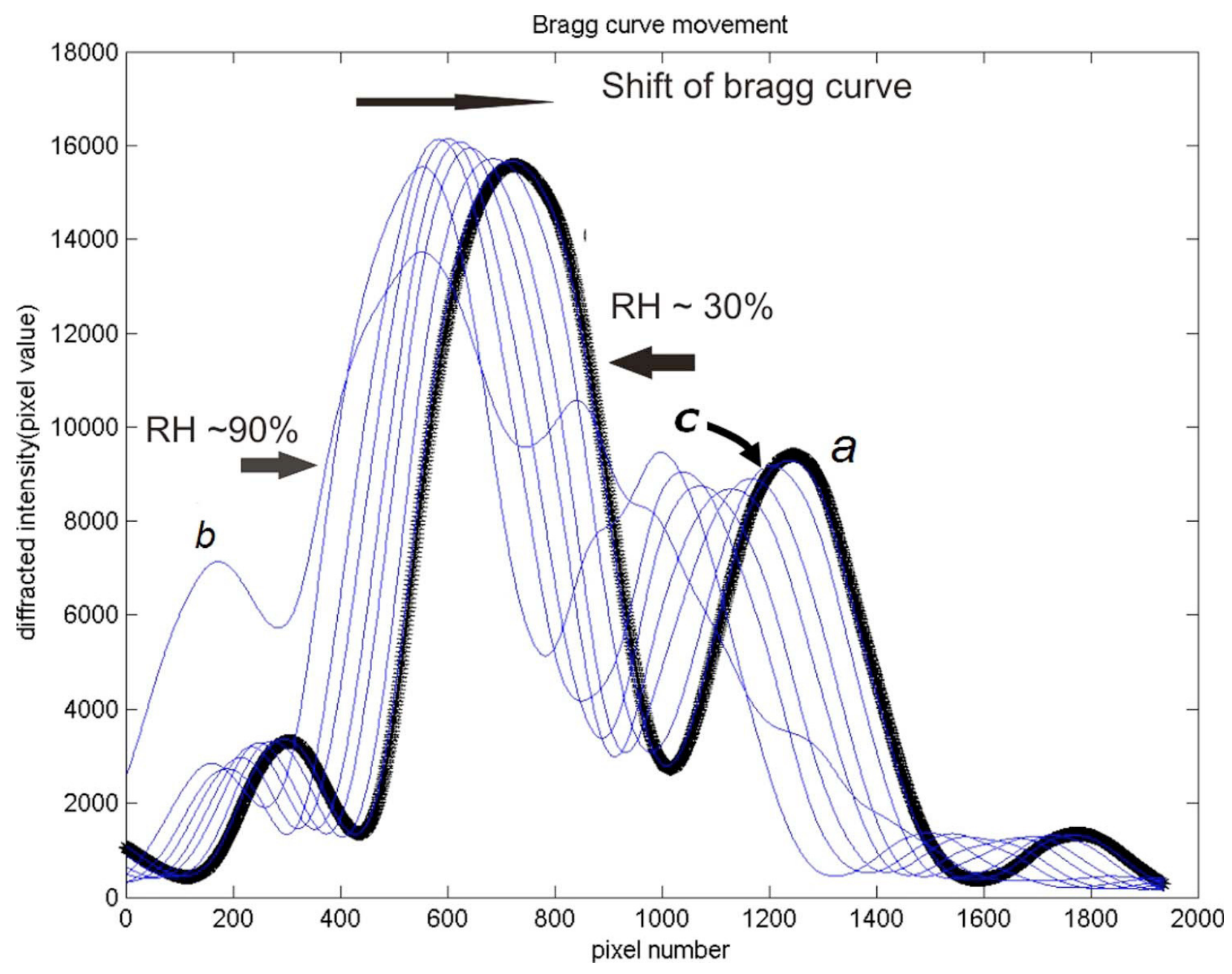

Fig 4. Observation of Bragg detuning of a grating when exposed to high humidity. The thickness of the grating changes with the environmental humidity. ' $a$ ' is the original profile. ' $b$ ' is the high humidity profile, ' $c$ ' is the last measured profile. 